Rhode Island College

Digital Commons @ RIC

\title{
Identifying Beliefs About Smoking in Patients with Peripheral Vascular Disease
}

Elizabeth Lopes-Costa

Rhode Island College

Follow this and additional works at: https://digitalcommons.ric.edu/etd

Part of the Nursing Commons

\section{Recommended Citation}

Lopes-Costa, Elizabeth, "Identifying Beliefs About Smoking in Patients with Peripheral Vascular Disease" (2016). Master's Theses, Dissertations, Graduate Research and Major Papers Overview. 148.

https://digitalcommons.ric.edu/etd/148

This Major Paper is brought to you for free and open access by the Master's Theses, Dissertations, Graduate Research and Major Papers at Digital Commons @ RIC. It has been accepted for inclusion in Master's Theses, Dissertations, Graduate Research and Major Papers Overview by an authorized administrator of Digital Commons @ RIC. For more information, please contact digitalcommons@ric.edu. 
IDENTIFYING BELIEFS ABOUT SMOKING IN PATIENTS WITH PERIPHERAL VASCULAR DISEASE

by

Elizabeth Lopes-Costa, RN, BSN

A Major Paper Submitted in Partial Fulfillment

of the Requirements for the Degree of

Master of Science in Nursing

in

The School of Nursing

Rhode Island College

2016 


\begin{abstract}
Peripheral vascular disease (PVD) is a chronic disabling illness that frequently results in the occlusion of arteries in lower limbs and may cause ischemia, gangrene, or infection. The single most significant factor contributing to both the onset and progression of PVD is smoking, which has addictive properties. Once smoking becomes a habitual behavior, combined with the atherosclerotic process, a disastrous pathway ensues. The aim of this project was to gain an understanding of the beliefs of patients with PVD who smoked. Data were obtained from a sample of participants with a known history of PVD who have had a previous vascular intervention and who are established patients in a vascular surgeon's private office where $\mathrm{N}=50$. The majority of participants were male $(n=31$; $63 \%) 65-74$ years of age $(n=18 ; 36 \%)$, were unemployed $(n=30 ; 60 \%)$ and have smoked for greater than $51+$ years $(n=13 ; 26 \%)$. A scale that was developed guided by The Behavioral beliefs subscale revealed that PVD patients enjoyed smoking, smoking helped them to relax, and that they believed that they were addicted to smoking. Results from the normative beliefs indicated that the spouse or significant other, children or those whom they lived with, did not approve of their smoking and also that their healthcare provider had discussed smoking cessation with them. The control beliefs subscale results demonstrated that most patients had attempted to quit smoking, believed it would be a difficult behavior to stop, believed that they would require the assistance of a patch or medication and feared the withdrawal symptoms. Exploring the beliefs and thought process of patients who smoked provided a rich body of knowledge which can afford the healthcare professional with the ability to better understand the smoking experience as seen through patients' eyes.
\end{abstract}




\section{Acknowledgements}

I would like to thank the following individuals for helping me through this program:

To my son Kaden, forgive me for spending so much of our precious time studying in order to give us a better life.

To my dad Joe, who was so happy to see me begin this, but unfortunately could not see me finish. Where ever he is now, may he be proud and watch over me.

To my Italian mom Elaine, for countless hours of help with this paper/program and words of encouragement.

To my best friend EJM, thank you for all sorts of reasons, far too many to mention.

And to my almighty creator, may he lead me down the path of happiness and success. 


\section{Table of Contents}

Background/Statement of the Problem ...........................................................................

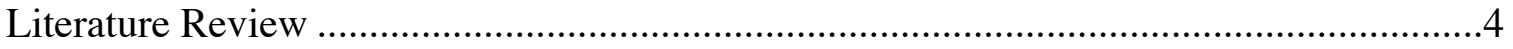

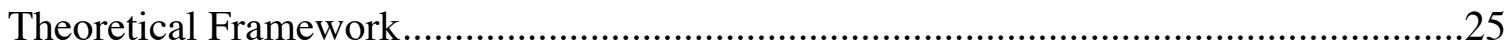

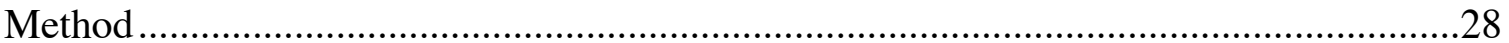

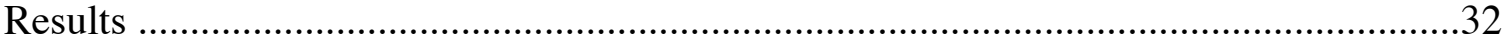

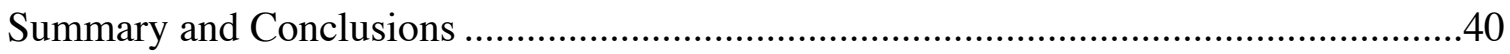

Recommendations and Implications for Advanced Nursing Practice ............................44

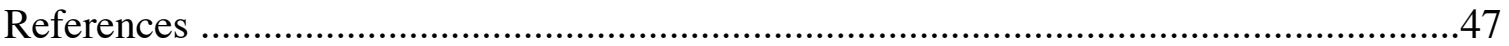




\section{IDENTIFYING BELIEFS ABOUT SMOKING IN PATIENTS WITH PERIPHERAL VASCULAR DISEASE \\ Background/Statement of the Problem}

Peripheral vascular disease (PVD), also known as arteriosclerosis obliterans, refers to the occlusion or stenosis of arteries, usually occurring in the lower extremities. This devastating disease can lead to loss of limb or even death. Between $5 \%$ and $10 \%$ of Americans, who are 40 years of age or older, are affected with PVD; of those, $40 \%$ are smokers. (Hennrikus et al., 2010) In these atherosclerotic arteries, a cholesterol/protein core called an atheroma gradually enlarges stenosing or occluding medium and large vessels. If the diameter of the artery is reduced by $50 \%$, this can lead to alterations in distal pressures afforded to affected muscle groups (Hennrikus et al., 2010). This ischemia results from atherothrombosis of the distal aorta and it's branching, causing intermittent claudication, rest pain, skin ulcerations, and even gangrene. (Creager, Kaufman, \& Conte, 2012)

The symptoms of PVD can appear as early as the patient's fifth decade of life, but usually do not become problematic until the patient experiences difficulty in ambulation or exercise intolerance (Hughson, Mann, \& Garrod, 1978). Typically, while at rest, blood flow is normal. At the onset of the disease process, these symptoms appear while ambulating for some distance, climbing stairs, or even with mild exercise. The proximal stenosis caused by the atherosclerotic process results in the affected arteries' inability to meet the metabolic blood demands of the distal muscles, subsequently leading to tissue ischemia manifesting in a cramping muscle pain (Powell, 1998). When the patient rests and ceases exercise, blood supply reaches muscles and pain then subsides. Peripheral vascular disease left untreated can severely debilitate a patient, and in some cases cause life threatening infections.

Numerous risk factors can affect atherosclerosis. Many of these factors can be

modified, others cannot. Aging and family history of early heart disease are not 
modifiable risk factors. Hypertension, obesity, diabetes, hyperlipidemia, sedentary lifestyle, and significantly smoking are risk factors that can be controlled or prevented. In this respect, smoking is both the disease and the cause of the disease (Powell, 1998).

In 1965, reports of the Surgeon General mandated Congress to require all cigarette packages distributed in the United States carry a health warning label. Consequently, by September of 1970 cigarette advertising was banned on television and radio. The anti-smoking campaign had begun. Almost 50 years later, society is well aware of the dangers of smoking. Most individuals can associate the use of tobacco with the development of cancer. Some individuals are concerned and quit, while others hear the warning and continue to smoke. Of particular interest are vascular patients who often appear to be unaware of the correlation between nicotine and atherosclerosis. Familiar anti-smoking campaigns in the media have traditionally focused on smoking's carcinogenic affects and not the destruction of the patients' vasculature.

Countless research studies are available regarding nicotine use and atherosclerosis. This disastrous combination is often what brings the vascular patient to seek a surgical intervention due to the onset of claudicant pain. Lack of recognition of this association between smoking and arterial stenosis can lead to repeated surgical interventions. This manifestation requires exploration due to the fact that for some individuals, even after repeated vascular interventions and multiple return visits to the vascular surgeon's office, the smoking habit continues. The key question to address is: what are the driving factors that cause a vascular patient to continue to smoke- habit, addiction, peer pressure, or the smoking experience itself?

The purpose of this project is to identify the behavioral, normative, control beliefs of patients with PVD toward smoking. Next, the review of the literature will be presented. 


\section{Literature Review}

Articles related to patient smoking and vascular disease were obtained from searches in PubMed, CINHAL, and Cochrane databases using the free text phrase "nicotine use and atherosclerosis", which resulted in over 200 articles. Additional searches using the MeSH term "vascular patient and smoking", "nicotine", "atherosclerosis", and "smoking cessation" returned several relevant results. In addition, the Theory of Reasoned Action (TRA) and the Theory of Planned Behavior (TPB) were

explored using the mentioned data bases and resulted in numerous research articles on application of the TRA/TPB to smoking behavior.

\section{Epidemiology of Smoking}


According to the World Health Organization (WHO) estimates, there are 1.1 billion tobacco smokers worldwide, constituting one third of the adult population. The majorities of smokers are seen in developed countries and are estimated at 800 million. The vast majority of smokers are male (67\%), with females constituting $33 \%$ (Fagerstrom, 2002). World-wide, tobacco use has resulted in more than 5 million adult deaths annually, with the largest numbers seen in the United States and Europe (Fagerstrom).

Another recent trend is the increased numbers of young smokers. In 1992, in the United States, $17 \%$ of all high school seniors smoked, while this number increased to $22 \%$ by 1995 . Assuming these staggering numbers will continue to rise, it is estimated that by 2030, worldwide smokers will increase to 1.6 billion, with a corresponding increase in tobacco related diseases and deaths (Fagerstrom, 2002).

Weinberger et al. (2014) performed a longitudinal investigation of smoking status that demonstrated an annual average of 480,000 tobacco-related deaths in the United States. This number has increased steadily in the last 50 years. With these staggering statistics, tobacco is predicted to be the leading cause of death by 2020 , resulting in one in every eight deaths. Smokers will die prematurely secondary to tobacco-use related diseases.

State-specific prevalence of current smoking surveyed in 2013 revealed that $20 \%$ of Rhode Island (RI) residents smoke. This number decreased slightly in 2013 to $17.4 \%$, but the increased number of smokeless tobacco use increased by $1.4 \%$, which offsets this data (Nguyen, Marshall, Hu, \& Neff, 2015).

The Center for Disease Control (CDC) reported that only one in seven individual's attempted quitting smoking during a 3-year period. The stability of the smoking rate is not related to the smokers desire to quit or a lack of smoking cessation attempts; the majority of cessation attempts end in relapse (Weinberger, Pilver, Mazure, \& McKee, 2014). 


\section{Hazards of Smoking}

Numerous research articles have been published on the hazards of cigarette smoking. The association between smoking and premature death has been established (Powell 1998). Varying complications result from smoking such as lung cancer, respiratory disease, cardiovascular complications, as well as peripheral atherosclerosis. Powell discussed vascular damage of the arterial wall from smoking and how nicotine specifically alters the cellular DNA. She focused on three separate stages of how smoking potentiates the development of atherosclerosis: endothelial dysfunction, monocyte recruitment and development of plaque, and thrombus at the damages of the arterial intima.

The combustion of tobacco in cigarettes releases hundreds of different chemicals including aromatic hydrocarbons, aromatic amines, carbon monoxide, nitrogen oxides, and nicotine. These hazardous chemicals interfere with a variety of cellular and metabolic process. Of these chemicals, nicotine has the most additive properties (Powell).

Ambrose and Barua (2004) summarized the pathophysiology of cigarette smoking and cardiovascular disease. They extensively elaborated on the cause of endothelial dysfunction of smoking to acute clinical events. The results connected cigarette smoking, more specifically, the exposure of nicotine, which predisposes individuals to several different atherosclerotic syndromes, coronary artery disease, and stroke, including aortic and peripheral atherosclerosis leading to intermittent claudication and abdominal aortic aneurysms.

Ambrose and Barua also examined clinical and experimental observation between cigarette smoking and thrombosis. In pathologic studies of sudden coronary death, cigarette smoking increased the risk of plaque rupture and acute thrombosis. Platelets isolated from smokers also revealed stimulated spontaneous aggregation and adhesion, proving platelet dysfunction. Smokers have higher fibrinogen levels altering tissue factor 
pathways, increasing thrombotic potential. Nicotine also affects substance-P-stimulated t-PA. This alteration of fibrinolysis causes dysfunctional thrombohemostatic mechanisms that promote the initiation and propagation of thrombus formation and inhibits its effective dissolution (Ambrose \& Barua).

In addition, reviewing the nicotine effects of atherosclerotic changes in a vessel, Ambrose and Barua correlated the association of packs smoked per year with the severity of atherosclerosis. The results indicated that either passive or active exposure to nicotine promoted vasomotor dysfunction, atherogenesis, and thrombosis in multiple vascular beds. The authors concluded that even though the association between cigarette smoking and cardiovascular risk are extensively documented, the linear dose effect is unclear. It was previously thought that the heavier the smoker, the higher cardiovascular risks. Newer data is suggestive of a non-specific dose-dependent correlation between heavy and light smokers (Ambrose \& Barua). Information now has been presented that supports that the underlying biochemical and cellular processes may become saturated even with a small amount of toxic components of smoking (Ambrose \& Barua).

\section{Nicotine Addiction}

Hatsukami, Stead, and Gupta (2008) conducted a seminar which focused on existing meta-analyses on treatments for smoking cessation and additionally, undertook a Medline search for articles related to drug treatments which were not included in the meta-analyses. Using Medline, the authors applied the following search terms: "smoking cessation", "tobacco cessation", "nicotine replacement therapy", and "antidepressants" among other several other terms to provide a comprehensive search. Only studies with sufficient sample size and rigorous methodology were selected as additional studies. The seminar provided descriptions for the diagnosis of tobacco addiction, the biobehavioral basis for addiction and evidence based treatments

Hatsukami et al. reviewed the criteria for nicotine dependence as described in the Diagnostic and Statistical Manual IV (DSM-IV) and the International Classification of 
Diseases, 10th revision (IDC-10). The DSM-IV and ICD-10 have been shown to have little predictive validity regarding relapse to smoking (Hatsukami et al.). The most widely used dependence measure is the Fagerstrom tolerance questionnaire. The evidence suggested regardless of diagnostic criteria for nicotine dependence, all smokers should be provided with treatment for smoking cessation (Hatsukami et al.).

The authors also reviewed the biobehavioral basis for addiction and found the physician and other health care professionals have a powerful effect in helping smokers to stop using tobacco. Although over $45 \%$ of smokers will try to quit on their own yearly, less than 5\% of them succeed (Hatsukami et al.). Physicians and other healthcare professionals can have a powerful effect to enhance smoking cessation rates by as much as five-fold. The authors found evidence that supported even just brief advice by healthcare professionals increased $(2.5 \%)$ absolute rate of smoking cessation compared to no advice. On average, in the USA about $70 \%$ of smokers seek healthcare and the authors suggested that this would be an excellent opportunity to provide effective treatments during office time. Also, counseling frequently involves education regarding the beneficial health effects of smoking cessation, as well as, problem-solving, skills training and social support are important but require additional factors. The use of a telephone quit line has proven beneficial when support is unavailable (Hatsukami et al.).

Hatsukami et al. reviewed evidence-based treatments, including pharmacotherapies. Nicotine replacement therapy (NRT) such as gum, lozenges, patches or sublingual tablets revealed positive results. In RCTs comparing nicotine replacement therapies (NRTs) versus placebo, a 12-week continuous abstinence rate varied from 20\%24\%. The mean quit rate at the six month mark for non-prescription NRTs was $7 \%$. The authors also performed an analysis to include bupropion and varenicline, two nonnicotine-based drugs also used as anti-depressants. These medications function as noncompetitive antagonist of the nicotinic acetylcholine receptor by blocking the reuptake of dopamine and noradrenaline. Varenicline is a potent alpha-4 beta-2 partial agonist, 
which provides tobacco withdrawal relief by its agonist action and blocks the reinforcing effects of nicotine by its antagonist action. Literature regarding combined therapies was also evaluated. Nicotine replacement therapies provide a steady concentration of nicotine throughout the day and bupropion/varenicline provides tobacco withdrawal relief. In these studies, smoking abstinence rates were $23 \%$ at six months when NRTs were combined with an anti-depressant, as opposed to only $10 \%$ of abstinence rates when NRTs were used alone. These numbers only reflect short-term abstinence; long-term ratings only sustained a $4 \%$ margin in some studies (Hatsukami et al.).

Although available pharmacotherapies can improve success rates, the absolute success rate remains fairly low (20\%) (Hatsukami et al.). The authors expressed that nicotine/tobacco addiction should be treated as a chronic disorder and that relapse should be seen as a probable event. Treatment should be multi-therapy combining counseling with pharmacotherapy. The consensus of the additional studies reviewed by Hatsukami et al. was that development of a comprehensive treatment program, encompassing multifaceted treatments, is required to treat tobacco addiction.

Benowitz (2009) conducted a comprehensive review of the neurobiology of nicotine addiction and withdrawal, as well as the implications for nicotine addiction therapy. Included was a review of the pharmacology of nicotine, the addictive properties, smoking-induced disease and therapeutics with an emphasis on individuals' potential vulnerability to tobacco dependence. Benowitz noted that with repeated exposure to nicotine, tolerance, or neuroadaptation, developed to some, but not all, of the effects of nicotine. Neuroadaptation was associated with an increase in the number of nAChRs binding sites in the brain, which are believed to play a role in tolerance and dependence. Smokers unknowingly continue to smoke throughout the day to maintain plasma levels and prevent withdrawal symptoms (Benowitz). These withdrawal symptoms are associated with negative emotional states and may include anxiety and 
perceived increased stress levels. These represent a powerful stimulus to relapse to tobacco use (Benowitz).

Benowitz (2009) described conditioned behavior and nicotine. The author stated that all drug-taking behavior is learned, as a result of conditioning. This behavior is reinforced by the pharmacologic actions of nicotine. Concurrently, as this reaction is occurring, smokers begin to associate certain moods or situations related to the reward effects of nicotine. The association between such cues and anticipated drug effects helps maintain the desire to smoke (Benowitz). People often smoke cigarettes in specific situations, like after a meal, with a cup of coffee or alcoholic beverage, or out with friends who also smoke. The association between smoking and these events repeated over time become powerful cues for the urge to smoke. Even unpleasant moods can become conditioned cues for smoking. A smoker may learn that not having a cigarette provokes irritability and smoking a cigarette provides relief (Benowitz).

Nicotine underlies addiction and sustains cigarette smoking; therefore considering nicotine replacement as a potential alternative to tobacco use for smokers who cannot quit may be an option (Benowitz, 2009). Nicotine replacement therapy has been shown to reduce smoking and increase smoking cessation rates. Nicotine as a substitute for the thousands of carcinogenic toxins in cigarettes can be beneficial, but with prolonged exposure to nicotine other risk factors such as, cardiovascular system effects can promote atherogenesis and precipitate acute ischemic events in patients with PVD (Benowitz).

Benowitz (2009) concluded that personalizing nicotine addiction treatment is the key to facilitating smokers to quit. Most smokers are aware of the dangers of smoking and how it can affect organs throughout the body. Quitting at any age may lead to significant reductions of smoking-related risk factors. Despite these facts, approximately $80 \%$ of smokers relapse within a few months of abstinence and only $3 \%$ remain abstinent three months out (Benowitz, 2009). 
In 2010, Benowitz examined nicotine addiction via an extensive review. Benowitz noted that 45 million Americans smoke, $70 \%$ of smokers would like to quit, $40 \%$ of smokers quit for one day. Eighty percent of those that do quit return to smoking within one month, with about $3 \%$ successfully quitting smoking. Benowitz found that when a smoker inhales smoke particles from a cigarette, nicotine enters the arterial circulation and moves in six seconds from lungs to brain and binds to nicotinic cholinergic receptors. Stimulation of these receptors then stimulates a variety of neurotransmitters in the brain. One of these is dopamine, an enzyme that triggers a pleasurable experience (Benowitz).

Benowitz (2010) concluded that nicotine sustains tobacco addition by inducing pleasure as well as reducing stress and anxiety. Smoking also has shown to improve concentration, reaction time, and enhance performances of certain tasks. Smoking cessation even for just a few hours causes withdrawal symptoms which commonly include irritability, depressed mood, restlessness, and anxiety. These withdrawals symptoms are often severe and to alleviate them smokers return to smoking. Smokers relate specific moods and cues to smoking. Understanding the mechanism can lead to the development of novel medication and smoking-cessation treatment (Benowitz).

\section{Current Strategies in Smoking Cessation}

Gorin and Heck (2004) performed a meta-analysis of research conducted between 1990 to 2004 using Medline, CINAH, PSYCINFO and dissertation abstracts related to the efficacy of tobacco counseling by health care providers. Over 200 articles were reviewed and of those 37 RCTs or quasi-experiments were selected. Since healthcare professionals can greatly influence patients' behavior, Gorin and Heck aimed to examine which providers were more effective: physicians, nurses, dentists, or multiprovider teams.

All RCTs or quasi-experiments utilized in-person counseling. Times varied from 3-5 minutes during a routine visit to structural behavioral change cessions lasting over an 
hour. Various counseling approaches were provided ranging from teaching patients to problem solve, relaxation methods, providing social support, education on the health dangers of smoking, or helping patients access intra-treatment or extra-treatment support.

Studies had a mean sample size of 507 clients, with 239 in the intervention arm and 268 in the control arm. Sixty-eight percent of the studies $(n=25)$ assigned patients to the intervention arm or control arm at random, although several $(n=32)$ assigned treatment by patient number or by using a quasi-experimental design. The majority of the studies $(76 \% ; n=28)$ recruited subjects from in an out-patient or hospital visit, and the remainder $(24 \% ; n=9)$ were in an inpatient setting. Ninety-seven percent $(n=36)$ of the interventions included counseling. One percent of the studies $(n=9)$ included mailed materials that were followed up with contact from a health care provider. Approximately half of the participants $(n=50)$ received follow-up by telephone, while the remainder $(n=50)$ were followed up during a clinical visit. The interventionists were divided by physicians (64\%), nurses (72\%), dentists (100\%) and team approached accounted for $64 \%$ of the studies. Data revealed that patients surveyed reported that between $34 \%-74 \%$ received cessation advice from their provider. Of these providers, $45 \%-85 \%$ were physicians, $36 \%-71 \%$ were nurses and $51 \%-61 \%$ were dentists.

Gorin and Heck concluded that receiving even limited form of advice from any healthcare provider produced a small increase in smoking cessation rates. Physicians $($ Effect Size $=6.01 ; 95 \%$ Confidence Interval, -2.46 to $13.29 ; \mathrm{P}=0.002)$ had higher smoking cessation rates, followed by dentists (Effect Size $=0.33 ; 95 \%$ Confidence Interval, -0.02 to $1.16 ; \mathrm{P}=0.12)$, then multi-provider teams $(\mathrm{ES}=0.79 \% ; 95 \% \mathrm{CI},-0.19$ to $3.71, \mathrm{P}=0.002)$, and finally, by nurses $(\mathrm{ES}=0.03 ; 95 \% \mathrm{CI},-0.30$ to $0.31 ; \mathrm{P}=0.37)$.

Wu et al. (2006) conducted a meta-analysis regarding the effectiveness of pharmacological smoking cessation therapies. The authors' primary outcome of interest was smoking cessation at one year. Findings included the following: Odds Ratio (OR) $1.71,95 \%$, Confidence Interval (CI) $1.55-1.88, \mathrm{P}=<0.0001$. Their secondary point of 
interest was smoking cessation at the three month interval. Wu et al. searched 10 reliable electronic data bases including but not limited to MEDLINE, EMBASE, CINAHL, TOXNET, and Cochrane. They conducted a meta-analysis of over 70 randomized controlled trials (RCTs) evaluating smoking cessation interventions at the one year mark. The inclusion criteria utilized only RCTs that used chemical confirmation as evidence of nicotine abstinence as an outcome. False reporting is most likely to occur in the trial setting or in assessing smoking status after a medical event. In both situations the smoker is under a considerable about of pressure to quit. Laboratory tests are, often used to verify smoking cessation, especially in verification in clinical trials. Only studies with the following chemical confirmation were utilized. Chemical confirmation included various methods of verifying smoking status including serum and saliva thiocyanate, expired carbon monoxide, plasma, saliva and urinary cotinine and nicotine. This data was not reported as it was simply used as a confirmation of smoking status.

Wu et al. (2006) also examined several RCTs regarding the use of pharmacological interventions. The three interventions included nicotine replacement therapy (NRT) (49 RCTs, OR 1.78, 95\% CI, 1.60-1.99), which was the most common; the use of anti-depressants such as bupropion (12 RCTs, OR 1.56, 95\% CI, 1.10-2.21, P = 0.01) and varenicline (4 RCTs, OR 2.96, 95\% CI, 2.12-4.12, $\mathrm{P}=<0.0001$ ); and the combination of NRTs and anti-depressants as co-therapies. The authors identified that effective mechanisms to improve smoking cessation are vital. Smoking is the number one preventable cause of death in the world. Cessation of smoking can also further reduce airway complication, lower rates of cerebrovascular disease, and coronary artery disease.

Wu et al. concluded that even though the exact mechanism of each intervention is different, using only one method for smoking cessation was not effective. At the one year mark smoking prevalence remained high among the experimental groups for all 3 
interventions. The authors suggested combination therapy should be individualized to the patient's symptoms.

A RCT by Hennrikus et al. (2010) studied the effectiveness of a smoking cessation program for peripheral vascular disease (PVD) patients. The authors' hypothesis was that patients with PVD need a focused intensive intervention, due to the serious nature of atherosclerotic disease which has the potential of causing major disability. Smoking is a completely modifiable risk factor affecting PVD. Outpatients with lower extremity PVD were identified from medical records as smokers. Subjects were randomly assigned to either an intensive PVD specific counseling intervention or a minimal smoking cessation intervention. The results suggested that subjects assigned to the intensive counseling were more likely to be confirmed abstinent from smoking at six month follow-up, with $21.3 \%$ in the intervention group vs. $6.8 \%$ in the minimal counseling group.

Tahiri et al. (2012) conducted a meta-analysis of RCTs of alternative smoking cessation therapies. The three alternatives to pharmacotherapies for smoking cessation were acupuncture, hypnotherapy, and aversive smoking. They systematically searched the Cochrane Library, EMBASE, Medline, and PsycINFO through December 2010. Only RCTs that reported cessation point outcome between six to 12 months were used. Of the articles searched 14 trials were selected: six investigated acupuncture, four investigated hypnotherapy and four investigated aversive smoking. A separate metaanalysis was carried out for each alternative therapy.

In respect to acupuncture, smoking cessation substantially increased (point estimate OR, 3.53). Hypnotherapy was found to be efficacious at promoting smoking cessation (point estimate OR 4.55); however, confidence intervals were wide thus limiting a strong conclusion. Aversive smoking substantially increased smoking abstinence compared with control (point estimate OR 4.26). All results were at the one year mark. 
Tahiri et al. concluded that up to $30 \%$ of individuals trying to quit smoking seek alternative interventions for fear of pharmacotherapies. The authors suggested that more healthcare providers offer these alternative smoking cessation aides to their patients and those researchers continue studying these methods for their efficacy.

\section{Attitudes and Beliefs About Smoking Cessation}

A dearth of literature exists on the attitudes and beliefs of patients and smoking cessation. Ronayne, O'Connor, and Scobie (1989) conducted a descriptive study which examined the differences in beliefs and social influences between patients who decided to quit smoking or decided to continue to smoke, following the diagnosis of PVD. Ronayne et al. sought to determine why some individuals, after learning of their PVD illness stop smoking, while the majority of individuals continue to smoke despite counseling from their healthcare provider. The authors investigated individuals with PVD, since the progression of the disease can lead to devastating disability or loss of limb. The beliefs influencing patients' decision to quit, reduce number of cigarettes, or to continue to smoke is not fully understood. The researchers conducted a study to determine the influence of beliefs and social influences on patients' decisions to quit or continue to smoking using Ajzen and Fishbein's Theory of Reasoned Action. A consecutive quota sample of 20 patients who had quit smoking and 22 smokers who continued smoking participated in the study. Data for this study were collected using a qualitative/quantitative methodology, utilizing the specific guidelines as described by Ajzen and Fishbein (1980). Each group was asked to complete a quantitative open ended survey questions using a 7-point Likert scale regarding questions about smoking or nonsmoking. The six quantitative beliefs about smoking outcomes listed in the survey were reduced risk of amputation, increased tension, weight gain, walk better, better circulation, and avoid leg surgery.

A qualitative analysis was performed looking for themes among the smokers and non-smokers. The three themes discussed were breathing better, walking better and the 
likelihood of amputation. Patients from both groups reported quitting smoking was more beneficial for their respiratory symptoms over their circulation symptoms. Patients reported "breathing better" and "coughing less". Patients were asked to indicate the likelihood of occurrence for a number of outcomes resulting from smokers to nonsmokers. Each outcome was rated on a 7-point Likert scale. Differences between smokers $(n=22)$ and non-smokers $(n=20)$ were significant on the outcomes of increased tension (non-smokers -0.79 , smokers $2.9, \mathrm{P}=0.003^{*}$ ), weight gain (non-smokers 1.1, smokers 3.4, $\mathrm{P}=0.01^{*}$ ), and walking better (non-smokers 4.3 , smokers $2.6, \mathrm{P}=0.035^{*}$ ). Non-smokers reported being more confident that smoking cessation would substantially increase the likelihood of walking better and reduce feelings of tension. The nonsmokers also reported not being fearful of weight gain since they had less difficulty walking.

The likelihood of amputation was elicited using probability scales of 0-100\%. Patients in both groups were asked the probability of amputation associated with quitting or continuing smoking. Attitudes towards smoking were divided into three categories: no risk (non-smokers 5.5\%, smokers 43\%), acceptable risk (non-smokers 0\%, smokers $10 \%$ ); and unacceptable risk (non-smokers 94\%, smokers 47\%). Patients who quit smoking reported the risk of amputation to be more substantially reduced than those who did not quit smoking. Attitudes toward the risk of amputation revealed 94\% $(n=18)$ of those who quit smoking perceived the risk of amputation as unacceptable while $53 \%$ $(n=11)$ of those who did not quit smoking perceived the risk to be negligible or acceptable. The remaining $47 \%(n=13)$ of smokers perceived the risk of amputation to be acceptable but were powerless to do anything about it. There were no statistically significant differences between smokers and non-smokers in demographic characteristics, clinical variables, and perceived social influences. Significant differences were found between groups in beliefs about and attitudes towards the risk of continued smoking. Of interest is the finding that respiratory issues (non-smokers $n=20$; smokers, $n=10$ ) 
provided more benefit over circulatory issues (non-smokers $n=8$; smokers, $n=4$ ). Both groups reported that their physician, family and friends encouraged them to quit smoking. The results of this study support the need to investigate more fully the factors which influence patients' decisions to quit or to continue to smoke.

Parry, Thomson, \& Fowkes (2001) examined the reasons for continuing to smoke from a sample of long-term older smokers who have PVD. The participants were a subset of a larger qualitative study of Life Course Influences on Patterns of Persistent Smoking. Semi-structured interviews were conducted. These studies enlisted the use of "life grids" which charted changes in levels and patterns of smoking against events and circumstance in the smokers' lives. It also examined the beliefs about why they smoke their current smoking behavior and their future intention. Some smokers justified continued smoking to the addictive properties of cigarettes, therefore, this addiction served to legitimize their claims of entitlement to the unhealthy habit. It appeared smokers feared the symptoms of nicotine deprivation, making quitting a difficult task.

The respondents $(n=70)$ were recruited from the Edinburgh Artery Study. The respondents were 22 current smokers and 48 ex-smokers, ages ranging from 65 to 84 years old, with at least 10 years smoking history, who averaged at least 10 cigarettes a week with presence of arterial disease. The study used quantitative interviews which incorporated life grids. A life grid is a system the authors utilized to collect patterns and levels of cigarette use across their life course. The grid examined smoking behaviors, including patterns of daily cigarette consumption, subjective dependence, and beliefs about social influence on smoking, the perceived relationship of life events/experiences and smoking, perceived influences, and advice from medical practitioner on smoking behavior.

The findings revealed older smokers with arterial disease defined their relationship to smoking in either a dependent or functional terms. Dependent smokers did not believe they were dependent on smoking while non-dependent smokers did not 
think of themselves as a smoker. Older smokers, despite high levels of cigarette consumption, made no correlation to addiction to justify their smoking. Others stated they "could quit at any time". These smokers perceived cigarettes as a positive resource; the perceived beliefs outweighed the benefits of quitting. Although Parry et al. examined the reasons to smoke their findings demonstrated their perceived beliefs outweigh benefits of quitting. Therefore, it is critical to identify patients' beliefs, normative beliefs, and control beliefs as a determinant of their behavior.

Clarke \& Aish (2002) conducted a descriptive cross-sectional aspect of a study of smokers with PVD who chose to participate in a smoking intervention compared with a group of smokers who declined to participate in the intervention. This study was based on the integration of the following three theoretical models: Ajzen and Fishbein's Theory of Reasoned Action, Kenney's Expected Utility Decision Theory, and Prochaska and DiClemete's Transtheoretical Model of Change. These theoretical models were used to describe the influence of this smoking cessation program on the beliefs and attitudes about smoking. Upon review of the extensive empirical studies related to smoking cessation, the authors noted patterns between smoking cessation and smokers' beliefs and attitudes, optimistic bias, and social influences. Certain individuals are more likely to quit smoking if they: believe that such actions will reduce the likelihood of disease progression, surgery, or amputation; perceive that smoking cessation is considered worthwhile by others they wish to please; consider living as non-smokers.

A nonprobability convenience sample was obtained from recruited participants who were smokers with vascular disease, at least 18 years or older, smoked at least one cigarette a day, were diagnosed with vascular disease, including PAD, carotid disease, and abdominal aortic aneurysms were where able to communicate in English. A smoking beliefs questionnaire designed to capture subjects' characteristics, stage of smoking change, subjective norm, attitudes, and expected utility was used. The participants completed the survey before the smoking cessation intervention and 13 weeks after the 
intervention. Smokers who decided not to participate in the intervention also completed a questionnaire for comparison. The intervention consisted of a physician guide with instructions about how to ask, advise, and assist patients to quit smoking, along with periodic assessment and personalized feedback reports.

The intervention group was identified as group 1 and included who participated in the smoking cessation program; group 2 included those who did not participate in the smoking cessation program but were willing to complete questionnaires. The results revealed statistically significant differences between group 1 and group 2 . Subjects in group $1(n=34,79 \%)$ were more often diagnosed with PAD than those in group $2(n=25$, $58 \%)$. Eighty-six percent $(n=37)$ of group 1 subjects smoked 11 or more cigarettes a day, whereas only $67 \%(n=28)$ of group 2 smoked 11 or more cigarettes per day. The amount smoked by the subjects in group 1 decreased significantly during the 13 week period from $86 \%(n=37)$ who smoked 11 or more cigarettes per day to only $58 \%(n=25)$. Eighty-one percent $(n=35)$ of group 1 believed that it was highly likely that they would develop more serious circulatory health problems, and only $50 \%(n=35)$ of group 2 believed this. Participants who did not enroll in the intervention were less educated (84\%, n=36 graduated high school in group 1 and 49\%, n=21 finished in group 2), less often diagnosed with PVD, were more in the precontemplating stage of change regarding smoking cessation and cared more about what the physician and family thought they should do. They perceived themselves to be at less risk for developing more severe circulatory problems if they did not stop smoking. This group of patients is on the disastrous pathway as described earlier.

When different populations were compared, there was no significant difference between primary care, in-patients, or pregnant women in the likelihood of quitting. What was significant were the providers who delivered the smoking cessation material. Physician were significantly more effective in promoting cessation $(\mathrm{Beta}=4.13, \mathrm{P}=$ 0.005). Nurses were less effective $(B e t a=-0.86, P=0.45)$. There were no significant 
differences between dentists and team providers ( $\mathrm{Beta}=0.73, \mathrm{P}=0.69)$. The findings support the promise of smoking cessation counseling by all healthcare providers and that additional longer-term trials of provider-based cessation approached are warranted.

A more recent study by Rajaee et al. (2014) examined whether the type of revascularization for peripheral arterial disease (PAD), percutaneous vs open procedure, was associated with smoking reduction. Among patients with PAD, smokers have a higher incidence of life- and limb-threatening complications, including lower extremity ischemic rest pain. Smokers are 2.3 times more likely to be symptomatic from PAD and have a higher incidence of lower extremity ischemic pain.

This study compared rates of smoking reduction after medical, percutaneous, and open intervention among patients with PAD and evaluated patients' perceptions of smoking. The study consisted of two parts, a retrospective chart review and a review of patients answered surveys. In the chart review, potential study participants were identified through a search of International Classification of Diseases, Ninth Division (ICD-9) codes for all patients seen by 1 of 3 vascular surgeons at an academic center. All patients undergoing evaluation and management for lower extremity PAD were included. Exclusion criteria included patients younger than 18 years old, absence of follow-up visits after initial consultation, and presence of acute limb-threatening ischemia.

The survey format allowed assessment of the patient perceptions of the impact that smoking had on the development of the disease. Patients were asked to rate several factors (diabetes, smoking, diet, sex, ethnicity, age, alcohol use, hypertension, and family history) based on the extent they believed the factor played a role in the development of disease. The surveys used a 5-point Likert scale to ask the participants whether their interventions were life-changing events and whether they had changed their diets, smoking habits, or attention to blood pressure as a result.

Of the 142 surveys submitted, 67 patients were former smokers, 20 were never smokers, and 55 were active smokers at the time of the intervention. Of the 67 former 
smokers, $25 \%(n=17)$ had undergone open interventions, $45 \%(n=30)$ had undergone percutaneous interventions, and 30\% $(n=20)$ had undergone medical management. None of the former smokers restarted smoking after their interventions. Of the 55 active smokers, 33\% $(n=18)$ had undergone open intervention, $44 \%(n=24)$ had undergone percutaneous interventions, and 22\% $(\mathrm{n}=12)$ had undergone medical management.

Rajaee et al. (2014) concluded that 55 patients were active smokers at the time of their intervention. Sixty seven percent $(n=8)$ in the medical management group, $50 \%$ $(n=12)$ in the percutaneous group, and $83 \%(n=15)$ in the open intervention group reduced their smoking by $50 \%$ after intervention. The authors reported that after controlling for several cofounders, open revascularization was independently associated with smoking reduction when compared to percutaneous intervention by $50 \%$. Surveys also revealed that $94 \%(n=23)$ of the participants believed that smoking was a significant contributor to their PAD. Patients with PAD who underwent open vascularization were more likely to reduce smoking by $50 \%$ compared to $(95 \% \mathrm{CI}, 1.18,76.67 ; \mathrm{P}=0.043)$ those who undergo percutaneous revascularization and that the perioperative period provide an opportunity to improve rates of smoking reduction (Rajaee et al., 2014).

Of the 55 participates included in the study, eighty three percent $(n=45)$ patients attempted smoking cessation (Rajaee et al., 2014). No established program for cessation existed at this institution, but the vascular surgeons routinely with each office visit offer smoking cessation counseling and discuss the benefits of quitting. For patients that are amenable to pharmacologic aids, either the vascular surgeons prescribed Chantix, nicotine patches, or nicotine gum. Or they referred the patients to their primary care provider for their prescriptions. A total of $37 \%(n=20)$ received a prescription for pharmacological aids, along with $37 \%(n=20)$ incorporated nicotine replacement therapy as well. Also $18.5 \%(\mathrm{n}=10)$ patients were referred for formal smoking cessation counseling, while $14.8 \%(\mathrm{n}=8)$ attempted hypnosis as a method to help them quit smoking. 
In summary, a comprehensive review of literature examined several areas for APRN and health care provider intervention: epidemiology of smoking; hazards of smoking; nicotine addiction; current strategies in smoking cessation; and more specific to this study, peoples' attitudes and beliefs about smoking cessation. There is a vast amount of literature regarding interventions pertaining to smoking cessation, however only four articles in the last 20 years examined patients' attitudes and beliefs towards smoking. The purpose of this study is to identify the attitudes and beliefs of patients with PVD. If attitudes and beliefs towards smoking can be identified, pertinent interventions may be developed in an effort to enhance smoking cessation efforts and in the long-term potentially reduce vascular interventions associated with smoking.

The next section describes the theoretical framework used to guide this study.

\section{Theoretical Framework}

The Theory of Reasoned Action (TRA) was first introduced by Ajzen and Fishbein in 1980. This model was developed for the prediction of behavioral intention, as a determinant of attitude and subjective norms. Ajzen and Fishbein posit that behavioral intention, as immediate antecedents to behavior are salient beliefs about the likelihood that preforming a particular behavior will lead to a specific outcome. (Madden \& Ellen, 1992) Behavioral intentions are divided into two salient beliefs: behavioral and normative. Ajzen stated the subsequent separation of behavioral intention from the behavior allows for explanation of limiting factors on attitudinal influence and subjective norm. In 1991, Ajzen published more articles extensively on TRA and then went on to extend the theory and developed the Theory of Planned Behavior (TPB) (Anderson \& 
Lavallee, 2008) The TPB extends the TRA with the addition of perceived behavioral beliefs and perceived power to predict deliberate behavior and how that behavior can be changed in individuals. According to TPB, human action is guided by three kinds of salient beliefs:

Behavioral beliefs: beliefs about the likely consequences of the behavior Normative beliefs: beliefs about the normative expectations of others Control beliefs: beliefs about the presence of factors that may facilitate or impede performance of the behavior. (Anderson \& Lavallee, 2008)

Figure 1. Theory of Reasoned Action and Theory of Planned Behavior

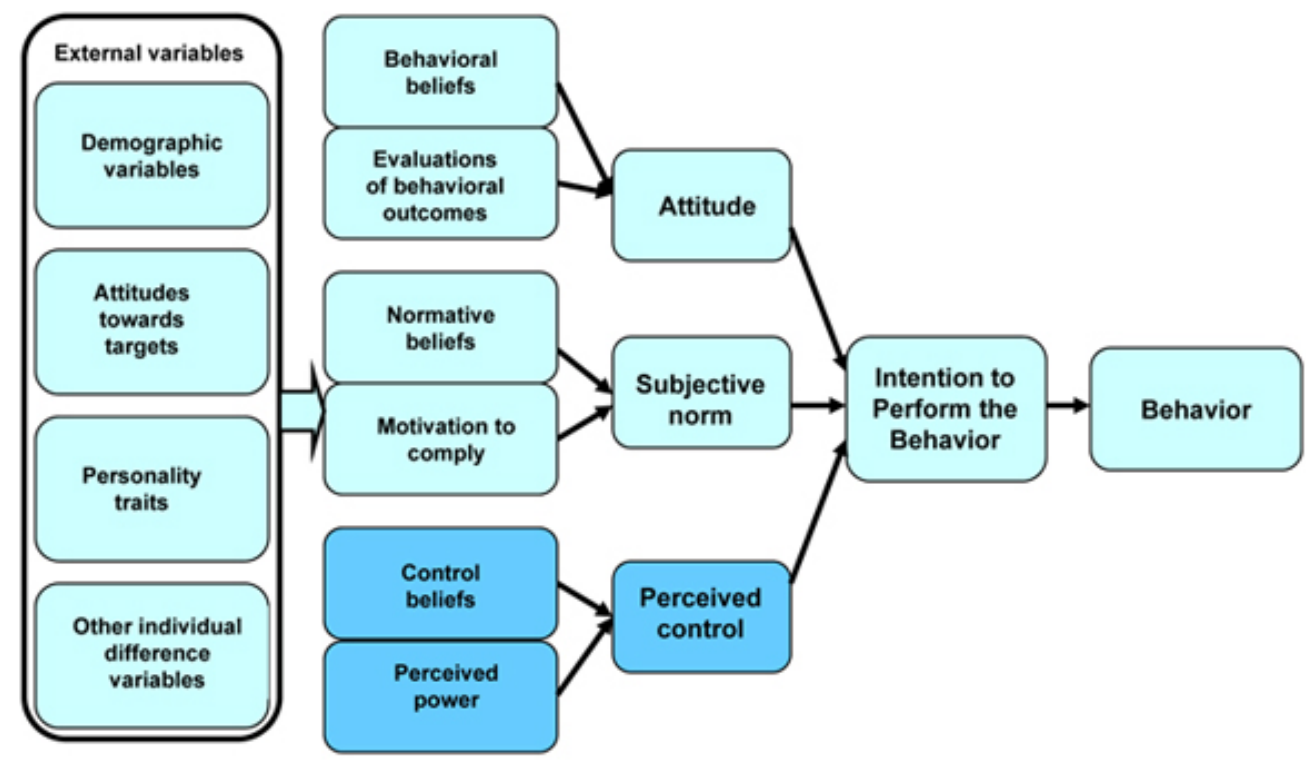

\section{Each behavior is defined within: Action, Target, Context, Time}

Note: Upper light area shows the Theory of Reasoned Action; entire figure shows the Theory of Planned Behavior

Ajzen's considerations are crucial in circumstances, projects, and programs that work with changing behaviors of people. In each perspective aggregate, behavioral beliefs produce a favorable or unfavorable attitude towards the specific behavior. Normative beliefs result in perceived social pressure and subjective norm. Control beliefs are the third antecedent of behavioral intention defined as the individual's belief concerning perception control and person's actual control over the behavior. 
The TRA and TPB are two of the most predictive persuasion theories in the literature. (Anderson \& Lavallee, 2008) These two theories have been applied to studies of the relationship among beliefs, attitudes, behavioral intentions, and behaviors in various arenas ranging from advertising to healthcare. Ajzen states that salient beliefs, normative beliefs, and control beliefs are components that lead to the formation of behavioral intent. In concert with one another, these beliefs are presumed to not only affect behavior directly, but also affect it indirectly through behavioral intention. Ajzen stated "If the individual has limited control of a given behavior, investigators should not only study the intention, but also the perceived control the individual has over the behavior" (Godin, Valois, Lepage, \& Deshamais, 1992, page 3). He further emphasized that perceived control can influence intention, as well as attitudes and subjective norm, it can predict behavior directly, in parallel with potential influence of intention, especially in situations where behavior is not under the control of the individual (Godin, Valois, Lepage, \& Desharnais, 1992).

Next, the methods in the study will be presented. 


\section{Method}

\section{Purpose}

The purpose of this project was to identify the behavioral, normative and control beliefs of patients with PVD towards smoking.

\section{Research question}

What are the behavioral, normative, and control beliefs of patients with PVD towards smoking?

\section{Design}

A non-experimental, descriptive design was utilized.

\section{Sample and Site}

A convenience sample was drawn from 50 patients at a vascular surgeon's private office associated with a major trauma center. Inclusion criteria were: age 65 or greater; must be a current smoker; who smoke at least one pack per day and have smoked for the past 15 years; had at least one vascular intervention, either bypass or stent. Exclusion criteria included: age 65 or less; do not currently smoke; have quit smoking for more than one year; never had a vascular intervention.

\section{Procedures}

Institutional Review Board (IRB) approvals were obtained from Rhode Island Hospital and Rhode Island College. Data collection began after IRB approval notification. The research investigator selected to focus the project around a vascular surgeon with high volume of patients with recurrent vascular disease. The research investigator was present in the waiting room of the vascular surgeon's office on the two days per week that consultations were provided. Upon arrival to office, all patients were routinely asked by the office staff to complete a CMS Medicare Payment Reconciliation System (PRS) Form for which addresses 4 questions: 1). Smoking status; 2). Pain scale; 3). Medications; 4). Surgical history. The potential participant's chart was pre-screened by the staff in the form of Medicare Inquiry of Smoking status. Either the patient's nurse 
or the doctor asked the patient if the NP student could speak to them about participating in a smoking survey study. The patient was approached by the physician in a private room and asked if he/she might be interested in participating in this study. The patient was given ample time to consider participation and a chance to ask any questions. If they agreed, detailed instructions regarding the purpose of the survey, and how to complete the survey, were provided. Potential participants were then provided the consent to review and questions were answered. Upon signed consent, participants were asked to complete the questionnaire before leaving the vascular surgeon's office. Although IRB approval has been obtained, all information collected was de-identified to ensure patient confidentiality.

\section{Measurement/Questionnaire Development}

The survey used collected the following demographic information: age; gender; years smoked; number of previous vascular surgeries/procedures; date of last procedure/surgery; level of education; and employment status. The purpose of this project was to identify the behavioral, normative and control beliefs of patients with PVD towards smoking.

In concert with this aim, the Theory of Planned Behavior (TPB) provides specific guidelines for researchers to adapt and develop their questionnaire to collect specific data. The Ajzen and Fishbein questionnaire guidelines were used and modified to capture the constructs of the TPB related to smoking. Using this guide each question was adapted to represent one of the three constructs as they related to smoking and PVD. Each question looked at behavioral, normative, or control beliefs as they related to each patients' thoughts toward smoking and how they felt smoking affected or didn't affect their PVD. The term questionnaire, which Ajzen utilizes, and survey will be used interchangeably.

A questionnaire was developed to obtain the required data utilizing the TPB. The questions developed for the questionnaire followed the TPB framework, appraising 
behavioral beliefs, normative beliefs (significant other influence) and control beliefs. The guidelines to develop the questionnaire define the behavior of interest in terms of its Target, Action, Context and Time. To secure reliable, internally consistent measures, it is necessary to select appropriate items in the formative stages of the investigation. (Ajzen, 2006) Therefore, selected items for the questionnaire were formulated to obtain a direct measure of behavioral beliefs, normative beliefs or perceived control beliefs toward the smoking behavior. The literature observations and clinical experience with patients with PVD regarding smoking were utilized to develop the items in the questionnaire. Specific items were developed to obtain the data required for each of the three belief sections to provide a complete summary of the patients thought process regarding smoking behavior.

The questionnaire was limited to two pages. Questions were closed-ended utilizing a Likert type rating scale ranging from 1-7 with bipolar adjectives that were anchored by 'not at all' (-) to 'very much' (+). Participant responses for each question were ranked on a Likert scale ranging from 1 to 7 with end points of "not at all" (-) to "very much" (+). The rankings were analyzed by the following scale where end point "not at all” utilized "extremely -“, "quite-“, and "slightly-“. The neutral or nonapplicable was represented by "neither". The end point of "very much" utilized "quite+", "slightly +", and "extremely+". Ordinal level data was collected. The font was at least size 14 to accommodate older participants or those with difficulties with smaller fonts. The questionnaire was developed at a sixth grade reading level.

The questionnaire was evaluated for its content validity by the two vascular surgeons. The physicians provided feedback on additional questions that should be included in the questionnaire. As a result, the following three questions were developed: Question 3.) Do you believe that smoking only causes cancer? Question 8.) Do you believe that your problem with blocked blood vessels is caused by smoking; and Question 9.) Do you believe that secondhand smoke can decrease blood flow in your legs? Each of the vascular surgeons were interested in their patients' thoughts on these 
three topics in an effort to obtain insight to some basic knowledge correlating nicotine and atherosclerosis.

\section{Data Analysis}

Demographics and belief questionnaire data were collected and managed using REDCap (Research Electronic Data Capture) tool hosted by Lifespan. REDcap is a secure, web-based application to support data capture for research studies, providing 1) an intuitive interface for validated data entry; 2) audit trails for tracking data manipulation and export procedures; 3 ) automated export procedures for seamless data downloads to common statistical package; and 4) procedures for importing data from external sources. Data analysis consisted of descriptive statistics of the demographic data: percents and means of the 20 item questionnaire were examined and exported to Microsoft Excel for further data analysis.

Next, the study results will be presented.

\section{Results}

\section{Demographics}

Table 1 on the next page illustrates the demographic data that were collected. The majority of the participants $(\mathrm{N}=50)$ were male $(\mathrm{n}=31 ; 67 \%)$ while females represented $37 \%(\mathrm{n}=19)$ of the sample. Age ranged from 25-85+ years; the age group 65-74 years represented $36 \%(n=18)$ of all participants while 55-64 years $30 \%(n=15)$ represented the second largest age category. Most participants had a high school education $(\mathrm{n}=21 ; 42 \%)$; two years of college/trade school $(n=17 ; 34 \%)$ ranked second. Most participants did not work $(n=30 ; 60 \%)$, while the remainder of the sample worked $(n=20 ; 40 \%)$. Smoking history ranged 11 years to 51+ years. Half of participants smoked for 41 to 51+ years $(n=24)$ or $50 \% ; 26 \%(n=13)$ smoked for $51+$ years or more, with $16 \%(n=8)$ in the $41-45$ years range. 
Table 1

Characteristics of Sample as Number and Percentage $(N=50)$

\begin{tabular}{|c|c|c|}
\hline Characteristic & $n$ & Percent \\
\hline \multicolumn{3}{|l|}{ Gender } \\
\hline Male & 31 & $63 \%$ \\
\hline Female & 18 & 37 \\
\hline \multicolumn{3}{|l|}{ Age Group } \\
\hline $25-34$ years & 1 & $2 \%$ \\
\hline $35-44$ & 3 & 6 \\
\hline $45-54$ & 8 & 16 \\
\hline $55-64$ & 15 & 30 \\
\hline $65-74$ & 18 & 36 \\
\hline $75-84$ & 3 & 6 \\
\hline $85+$ & 2 & 4 \\
\hline \multicolumn{3}{|l|}{ Education } \\
\hline Less than High School & 7 & $14 \%$ \\
\hline High School & 21 & 42 \\
\hline 2 years of College/Trade & 17 & 34 \\
\hline 4 years of College & 1 & 2 \\
\hline Graduate School & 4 & 8 \\
\hline \multicolumn{3}{|l|}{ Employment } \\
\hline Yes & 20 & $40 \%$ \\
\hline No & 30 & 60 \\
\hline \multicolumn{3}{|l|}{ Years Smoke } \\
\hline 11-15 Years & 1 & $2 \%$ \\
\hline $16-20$ & 3 & 6 \\
\hline $21-25$ & 5 & 10 \\
\hline $26-30$ & 4 & 8 \\
\hline $36-40$ & 7 & 14 \\
\hline $41-45$ & 8 & 16 \\
\hline $46-50$ & 3 & 6 \\
\hline $51+$ & 13 & 26 \\
\hline \multicolumn{3}{|c|}{ Number of Vascular Procedures and Surgeries } \\
\hline 1 & 16 & $32 \%$ \\
\hline 2 & 11 & 22 \\
\hline 3 & 13 & 26 \\
\hline 4 & 2 & 4 \\
\hline 5 & 1 & 2 \\
\hline 6 & 2 & 4 \\
\hline 10 & 2 & 4 \\
\hline 11 or greater & 3 & 6 \\
\hline \multicolumn{3}{|l|}{ Last Procedure } \\
\hline Less than 1 month & 5 & $10 \%$ \\
\hline More than 3 months & 6 & 12 \\
\hline More than 6 months & 10 & 20 \\
\hline More than 1 year & 20 & 40 \\
\hline More than 5 years & 5 & 10 \\
\hline 10 years + & 3 & 6 \\
\hline
\end{tabular}


Table 2 provides an overview of the individual questions and the number of participants that responded positively, negatively, or not at all (where $n=+$ are the participant responses that were read on the positive end of the scale while $n=-$ are participant responses that were read on the negative end of the scale).

\section{Table 2}

\section{Participant Responses Positive (+) Negative (-) Neither Rating}

\begin{tabular}{|c|c|c|c|c|c|}
\hline \multicolumn{2}{|c|}{ Behavioral, Normative and Control Beliefs } & Mean & $\mathrm{n}=+$ & $n=-$ & Neither \\
\hline \multicolumn{6}{|l|}{ Behavioral } \\
\hline Question 1 & How much do you enjoy smoking? & 4.88 & 29 & 13 & 8 \\
\hline Question 2 & Does smoking help you to relax? & 5.48 & 39 & 9 & 2 \\
\hline Question 3 & Do you believe that smoking only causes cancer? & 2.48 & 12 & 36 & 2 \\
\hline Question 4 & Do you believe you smoke because you are stressed? & 4.62 & 30 & 15 & 5 \\
\hline Question 5 & Do you believe you smoke because you are bored? & 4.08 & 26 & 20 & 4 \\
\hline Question 6 & $\begin{array}{l}\text { Do you believe that smoking helps to control your } \\
\text { weight? }\end{array}$ & $\begin{array}{l}2.76 \\
5.94\end{array}$ & $\begin{array}{l}13 \\
43\end{array}$ & $\begin{array}{l}31 \\
6\end{array}$ & $\begin{array}{l}6 \\
1\end{array}$ \\
\hline Question 7 & Do you believe that you are addicted to smoking? & & & & \\
\hline Question 8 & $\begin{array}{l}\text { Do you believe that your problem with } \\
\text { blocked blood vessels are caused by smoking? }\end{array}$ & $\begin{array}{l}4.76 \\
3.96\end{array}$ & $\begin{array}{l}31 \\
22\end{array}$ & $\begin{array}{l}13 \\
18\end{array}$ & $\begin{array}{l}6 \\
10\end{array}$ \\
\hline \multicolumn{2}{|r|}{ Do you believe second hand smoke can } & & & & \\
\hline \multicolumn{6}{|l|}{ Normative } \\
\hline \multirow[t]{2}{*}{ Question 10} & Does your spouse/significant other approve of & & & & \\
\hline & your smoking? & 2.86 & 7 & 28 & 15 \\
\hline Question 11 & $\begin{array}{l}\text { Do your children or those who live with you } \\
\text { approve of your smoking? }\end{array}$ & 2.42 & 4 & 31 & 15 \\
\hline \multirow{2}{*}{$\begin{array}{l}\text { Question 12 } \\
\text { Question 13 }\end{array}$} & Do your peers influence/support your smoking? & 2.40 & 5 & 35 & 10 \\
\hline & Has your healthcare provider talked about & 636 & 4 & 44 & \\
\hline Question 14 & How often are you around others who smoke? & 4.52 & 25 & 20 & 5 \\
\hline \multicolumn{2}{|c|}{ Control } & & & & \\
\hline \multicolumn{2}{|l|}{ Question 15} & 6.38 & 47 & 3 & 0 \\
\hline Question 16 & $\begin{array}{l}\text { Do you believe it would be easy not to smoke next } \\
\text { month? }\end{array}$ & 3.46 & 18 & 27 & 5 \\
\hline Question 17 & $\begin{array}{l}\text { How much control do you think you have over } \\
\text { whether you smoke? }\end{array}$ & $\begin{array}{l}4.22 \\
3.24\end{array}$ & $\begin{array}{l}22 \\
17\end{array}$ & $\begin{array}{l}21 \\
32\end{array}$ & $\begin{array}{l}7 \\
1\end{array}$ \\
\hline \multirow{2}{*}{$\begin{array}{l}\text { Question } 18 \\
\text { Question } 19\end{array}$} & Do you believe you can quit smoking without help? & & & & \\
\hline & $\begin{array}{l}\text { Do you believe you can quit smoking with the } \\
\text { help of a patch or medication? }\end{array}$ & 3.50 & 19 & 25 & 6 \\
\hline Question 20 & $\begin{array}{l}\text { Are you afraid of nicotine withdrawal symptoms } \\
\text { if you stop smoking? }\end{array}$ & 2.96 & 16 & 32 & 2 \\
\hline
\end{tabular}

The major findings of the Behavioral beliefs suggested that the majority of participants enjoyed smoking $(n=29)$ while $(n=8)$ were indecisive. Smoking is used to help participants to relax $(n=39)$ and most participants $(n=43)$ believed they are addicted to smoking. The effects of second hand smoke were neutral while $(n=10)$ were again 
indecisive. The major findings of the Normative beliefs were family, significant other, children and peers do not approve of the habit of smoking while $(n=15)$ had no opinion. The major findings of the Control beliefs were most participants $(n=47)$ tried to stop smoking in the past and feared the withdrawal symptoms $(n=32)$.

\section{Participants’ Behavioral Beliefs Reponses}

Table 3 provides an overview of the mean scores for each behavioral belief as listed in Questions 1-9 of the survey. Participant responses for each question were ranked on a Likert scale ranging from 1 to 7 with end points of "not at all" (-) to "very much" $(+)$. The rankings were analyzed by the following scale where end point "not at all" utilized "extremely -“, "quite-“, and "slightly-". The neutral or non-applicable was represented by "neither". The end point of "very much" utilized "quite+", "slightly +", and "extremely+".

\section{Table 3}

Participant Responses to Behavioral Beliefs

\begin{tabular}{ll}
\hline Behavioral Beliefs & Mean
\end{tabular}

1. How much do you enjoy smoking? 4.88

2. Does smoking help you to relax? $\quad 5.48$

3. Do you believe that smoking only causes cancer? 2.48

4. Do you believe you smoke because you are stressed? 4.62 
5. Do you believe you smoke because you are bored? $\quad 4.08$

6. Do you believe that smoking helps to control your weight? 2.76

7. Do you believe that you are addicted to smoking? 5.94

8. Do you believe that your problem with blocked blood vessels is caused by $\begin{array}{ll}\text { smoking? } & 4.76\end{array}$

9. Do you believe second hand smoke can decrease blood flow in your legs? 3.96

The mean score for behavioral beliefs ranged between 4.08 and 5.94, with the exception of question 3 ("Only smoking causes cancer"), question 6 ("Smoking helps to control your weight”), and question 9 ("Secondhand smoke can decrease blood flow in your legs"). The mean of 2.48 for question 3 indicated participants' belief that other diseases cause cancer, as well as smoking. The mean for question 6 was 2.76, which indicated smoking does not control weight. The mean of 3.96 for question 6 , secondhand smoke can decrease blood flow to your legs, indicated a lack of knowledge concerning the effects of second hand smoke.

\section{Participant Normative Beliefs Reponses}

Table 4 provides an overview of the mean scores for each normative belief as listed in questions 10 through 14. Participant responses for each question were ranked on a Likert scale ranging from 1 to 7 with end points of "not at all" (-) to "very much" (+). The rankings were analyzed by the following scale where end point "not at all" utilized "extremely -“, "quite-“, and "slightly-“". The neutral or non-applicable was represented by "neither". The end point of "very much" utilized "quite+", "slightly +", and "extremely+".

Table 4 
Participant Responses to Normative Beliefs

Normative Beliefs

Mean

10. Does your spouse/significant other approve of your smoking?

2.86

11. Do your children or those who live with you approve of your smoking?

2.42

12. Do your peers influence/support your smoking?

2.40

13. Has your healthcare provider talked about quitting smoking?

6.36

14. How often are you around others who smoke?

4.52

The mean scores ranged from 2.40-2.86. Spouse, significant other, children, those lived with, and peers all strongly opposed smoking behavior. Most notably, the highest mean score 6.36, question 4, which reflected the importance of the healthcare providers' recommendations on smoking cessation. Also, question 5, with a mean of 4.52 suggested participants with PVD are frequently around others who smoke.

\section{Participant Control Beliefs Reponses}

Table 5 provides an overview of the mean scores for each control belief as listed in questions 15 to 20. Participant responses for each question were ranked on a Likert scale ranging from 1 to 7 with end points of "not at all" (-) to "very much" (+). The rankings were analyzed by the following scale where end point "not at all" utilized "extremely -“, "quite-“, and "slightly-“. The neutral or non-applicable was represented by "neither". The end point of "very much" utilized "quite+", "slightly +", and "extremely+".

Table 5

Participant Responses to Control Beliefs

Control Beliefs

Mean 
15. Have you ever tried to quit smoking before? 6.38

16. Do you believe it would be easy not to smoke next month? 3.46

17. How much control do you think you have over whether you smoke? 4.22

18. Do you believe you can quit smoking without help? 3.24

19. Do you believe you can quit smoking with the help of a patch or medication? 3.50

20. Are you afraid of nicotine withdrawal symptoms if you stop smoking? 2.96

The mean scores ranged between 3.24-4.22 reflecting the belief that smoking cessation would be a difficult task. Participants' perception of the control they have to stop smoking varied from both endpoints with 'extremely' no control $(\mathrm{n}=21)$ to 'extremely' have control $(n=22)$ equally. In addition, most subjects indicated they needed help to quit smoking and required a patch or medication. Most patients, as reflected by the highest mean score of 6.38, attempted to quit smoking in the past and failed. The lowest mean score of 2.96 suggested patients with PVD did not want to experience smoking withdrawal symptoms.

In summary, the principle findings of this study suggest patients with PVD smoke because they find it pleasurable and want to quit but fear the adverse effects. Family and friends are important regarding this decision but fail to make a difference. Therefore, this data provides a compelling basis for future educational endeavors. Next, summary and conclusions are discussed. 


\section{Summary and Conclusions}

This research study originated from the researcher's many years of experience caring for patients with PVD. These patients often endure numerous readmissions to the hospital for revascularization interventions and despite that continue to smoke. It is imperative to explore the reason why such patients continue to smoke. One of the most compelling relevant studies in the literature was conducted by Ronayne, O'Connor, and Scobie (1989). This descriptive study examined the differences in beliefs and social influences between patients who decided to quit smoking or decided to continue to smoke, following the diagnosis of PVD. Ronayne et al. sought to determine why some individuals, after learning of their PVD illness, stop smoking while the majority of individuals continue to smoke despite counseling from their healthcare provider. The following six beliefs about smoking were elicited: increased tension; weight gain; walk better; better circulation; avoid leg surgery; and reduced risk of amputation,. However, an important finding was that patients believed their improved respiratory status was more beneficial than their improved peripheral vascular status. It was precisely this insight into patients' thought process that has guided the efforts of healthcare providers to establish smoking cessation regimens for individuals with PVD who continue to smoke. The purpose of this project was to identify the behavioral, normative and control beliefs of patients with PVD towards smoking. 
The TPB (Ajzen, 1985) was applied to smoking behavior to explore the three belief determinants of behavior. Behavioral beliefs produce a favorable or unfavorable attitude toward the smoking behavior; normative beliefs reflect the individual's beliefs regarding significant others' approval or disapproval of their smoking and result in perceived social pressure or subjective norm. Finally, control beliefs give rise to perceived behavioral control as a determinant of behavior. The three constructs of the TPB were incorporated into the survey developed by this researcher as specific questions, guided by the author's instructions.

A convenience sample was drawn from 50 participants at a vascular surgeon's private office associated with a major trauma center. The study participants consisted of both males and females. Of the 50 participants, the majority were male, age 65-75, unemployed, with either a high school or two years college/trade school education, who smoked for 40+ years, experienced one to three procedures/surgeries in the past, and where the last procedure occurred within one year. The participants completed a questionnaire that asked them to rate 20 questions about smoking on a Likert scale . In general, the behavioral belief questions suggested that patients with PVD find smoking pleasurable, while others responded negatively to this question and indicated they were disgusted with their smoking habits but were nevertheless addicted. Most participants responded that smoking helps them to relax. Similarly, participants also responded that they smoked because they were stressed and use smoking as an aid for stress reduction and relaxation. Participants responded equally that they smoked because they were bored or in contrast boredom had nothing to do with the smoking behavior, raising the issues of habit and addiction for this belief. Participants did not believe that only smoking causes cancer, which supports the rationale to smoke. This finding infers a belief that patients with PVD utilize smoking to support their smoking behavior. This is a defense mechanism to continue this risky behavior. Denial of the causal connection 
between smoking and cancer is evident as participants inferred that other diseases also cause cancer: Individuals who do not smoke get lung cancer as well.

Contrary to popular belief that smoking curbs appetite, this idea was not supported by the participant responses. Participants in this sample did not smoke to control their weight. Participants indicated that their blocked blood vessels were caused by smoking, however, they continued to smoke and experience harmful, physical effects. Participants were not aware that secondhand smoke can be harmful. Smoking's harmful effects occur via inhaling byproducts of smoke where secondhand smoke is in the atmosphere and has harmful effects to those exposed to it.

The normative belief questions revealed that the spouse or significant other did not approve of the participant smoking behavior. Children and peers as well did not approve of the smoking behavior. The principal finding was that the healthcare provider had discussed smoking cessation with the patient $(n=44 ; 88 \%)$. In light of this social pressure, people with documented PVD continued to smoke. The results of the frequency with which participants are around others who smoke did not offer any differences. Although the measure of subjective norm suggested disapproval by others of an undesirable behavior, patients continued to smoke.

The control belief questions suggested that a significant number of participants tried to quit smoking in the past $(n=47 ; 94 \%)$. Smoking cessation would not be an easy task to perform and perception of control was viewed both positively $(n=22 ; 44 \%)$ and negatively $(n=21,42 \%)$. Assistance may be required by some but most believed they could do it without a patch or medications and most participants worried about smoking withdrawal symptoms. Perceived behavioral control beliefs captured the participants' confidence that they are capable of performing the behavior under investigation.

This research study had several limitations. The sample was drawn from one private vascular physician's office where patients voluntarily sought care; therefore, the ability to generalize finding to the larger population is limited. Additionally, the focus of 
this study was on a specific patient population, patients with PVD who smoke. A methods limitation was the development of the belief questionnaire; a qualitative openended survey could have been administered to elicit what patients like and dislike about the behavior under study, in this case smoking. Subsequently, the beliefs which emerged from this qualitative data are then used to develop the quantitative scales which are then evaluated by the same patients. This would then apply to the other normative and control belief sections as well. Beliefs are not directly observed but must be inferred from observable responses to posed questions.

Next, recommendations and implications for advanced practice nursing will be discussed.

\section{Recommendations and Implications for Advanced Nursing Practice}

Morbidity related to smoking remains the leading preventative cause of death in the United Sates today. Research has shown that health promotion efforts tailored to the population are most effective and the advanced practice nurse (APRN) is in a unique position to deliver smoking cessation effectively. This study identified patients with PVD beliefs of smoking and its effect on their disease process, atherosclerosis. Previous 
research concluded that individuals with PVD continue to smoke without fear of repeated revascularization surgeries or even the threat of limb loss. Within the theoretical framework of the TPB, a patient's deliberate behavior can attempt to be predicted. The theory posits how attitudes, subjective norms, and control beliefs are in part determinants of volitional behavior.

The APRN is in a unique position to develop programs or interventions that optimize patient outcomes. This research, combined with the relevant available research, can provide a beginning foundation for program development. When working with patients, it is recommended that providers initially elicit their beliefs about the designated behavior change. Identifying beliefs can assist in gaining insight into the cognitive foundation of why patients behave in a particular manner. With this knowledge, the APRN can develop a smoking cessation program based on patient beliefs and an understanding of patients' behavior. Most educational programs or interventions are developed based upon what other healthcare professionals believe to be important to change behavior. However, Ajzen \& Fishbein have demonstrated in their numerous studies that beliefs are determinants of behavior. The APRN has the ability to effect change and is situated to provide nurse education and policy enhancement in the inpatient or community setting. It is frequently the role of the APRN to develop evidence-based policies within the healthcare system. Therefore, specific nursing interventions or program development derived from patient beliefs may be more likely to result in successful patient outcomes. More globally, implementing a research program of study based on what patients want to know or find useful can be the premise from which educational efforts are derived. 
This research revealed several opportunities for practice improvement. Examining beliefs of patients with PVD provides a greater understanding of what goes on in the thought processes of patients, their unique challenges, and the unaddressed needs they face. Exploring the patients' belief system provides insight into practice improvement regarding smoking cessation. The APRN is adept at identifying existing research and disseminating the findings to improve nursing practice. The role of the APRN is to role model evidence-based practice and improve patient outcomes. To accomplish this goal, the APRN should recognize their role as a colleague within the interdisciplinary practice team and their ability to serve as a team member and leader, drawing upon nursing's unique body of knowledge. The APRN is well suited to develop programs or interventions that encompass a holistic perspective of patient care and wellness promotion, both hallmarks of advanced practice nursing.

Nurse-driven research is needed to further explore why PVD patients continue to smoke while risking permanent disability. Further research is also indicated to examine the relationship between smoking and the extent of revascularization procedures.

The APRN is a clinical expert. Understanding what patients believe and their inner most thoughts are what constitutes clinical nursing knowledge. 


\section{References}

Ajzen, I. (1985). From intentions to actions: A theory of planned behavior. Springer Berlin Heidelberg, 11-39.

Ajzen, I. (2006). Theory of Planned Behavior Questionnaire. Retrieved from www.midss.ie

Ambrose, MD, FACC, J. H., \& Barua, MD, PhD, R. S. (2004). The Pathophysiology of Cigarette Smoking and Cardiovascular Disease. Retrieved from doi:10.1016/j.jacc.2003.12.047

Anderson, A. G., \& Lavallee, D. (2008). Applying the Theories of Reasoned Action and Planned Behavior to Athlete Training Adherence Behavior. Applied Psychology: An International Review, 57, 304-312.

Benowitz, MD, N. L. (2010). Nicotine Addiction. New England Journal of Medicine, 362 (24) Retrieved from doi:10.1056.1056/NEJMra0809890 
Benowitz, N. L. (2009). Pharmacology of Nicotine: Addiction, Smoking-Induced Disease, and Therapeutics. Annu Rev Pharmacol Toxicol, 57-71. http://dx.doi.org/10.1146/annurev.pharmtox.48.113006.094742

Clarke, HBScN, MScN, RN, K. E. \& Aish, BSN, MN, PhD, A. (2002). An exploration of health beliefs and attitudes of smokers with vascular disease who participate in or decline a smoking cessation program. Journal of Vascular Nursing, 20(3), 97105. http://dx.doi.org/10.1067/mvn.2002.127735

Creager, MD, M. A., Kaufman, MD, J. A., \& Conte, MD, M. S. (2012). Acute Limb Ischemia. Retrieved from NEJM.org

Fagerstrom, K. (2002). The Epidemiology of Smoking. Drugs, 62, 1-9. http://dx.doi.org/10.0012-6667/02/0002-0001/

Godin, G., Valois, P., Lepage, L., \& Desharnais, R. (1992). Predictors of smoking behaviour: an application of Ajzen's theory of planned behaviour. British Journal of Addiction, 87(3), 1335-1343. Retrieved from www.reasearchgate.net

Gorin, S. S., \& Heck, J. E. (2004, December 2004). Meta-Analysis of the Efficacy of Tobacco Counseling by Health Care Providers. Cancer Epidemiology, Biomarkers \& Prevention, 12(2012-2022), 2012-2022. Retrieved from cebp.aacrjournals.org

Hatsukami, PhD, D. K., Stead, MSc, L. F., \& Kupta, PhD, P. C. (2008, June 14, 2008). Tobacco Addiction. The Lancet, 371, 2027-2038. Retrieved from www.thelancet.com

Hennrikus, PhD, D., Joseph, MD, MPH, A. M., Lando, PhD, H. A., Duval, PhD, S., Ukestad, MS, L., Kodl, PhD, M., \& Hirsch, MD, A. T. (2010, July 6, 2010). Effectiveness of a Smoking Cessation Program for Peripheral Artery Disease Patients. Journal of the American College of Cardiology, 56(25), 2105-2112. http://dx.doi.org/10.1016/j.jacc.2010.07.031 
Hughson, W. G., Mann, J. I., \& Garrod, A. (1978, May 27, 1978). Intermittent claudication: prevalence and risk factors. $B M J, 1,1379-1381$. Retrieved from www.ncbi.nlm.nih.gov/

Madden, T. J., \& Ellen, P. S. (1992, February 1992). A Comparison of the Theory of Planned Behavior and the Theory of Reasoned Action. PSPB, 18(1), 3-9. http://dx.doi.org/10.1177/0146167292181001

Montano, D. E., \& Kasprzyk (2008). Theory of reason action, theory of panned behavior, and the integrated behavioral model. In K. Glanz, B. K. Rimer, \& K. Viswanath (Eds.), Heath Behavior and Health Education: Theory, Research, and Practice (4th ed., pp. 67-96). Retrieved from http://www.mdquit.org/health-behavior

Nguyen, MS, K., Marshall, MPH, L., Hu, DrPH, S., \& Neff, PhD, L. (2015, May 22, 2015). State-Specific Prevalence of Current Cigarette Smoking and Smokeless Tobacco Use Among Adults Aged >18 Years- United States, 2011-2013. Morbidity and Mortality Weekly Report, 64, 532-536. Retrieved from www.cdc.gov/mmwr/annual_data/2013

Parry, O., Thompson, C., \& Fowkes, F. R. (2001, April 18, 2001). Dependent behaviors and beliefs: a qualitative study of older long-term smokers with arterial disease. Addiction, 96(7), 1337-1347. http://dx.doi.org/10.1080/09652140120070391

Powell, J. T. (1998). Vascular damage from smoking: disease mechanisms at the arterial wall. Retrieved from http.//vmj.sagepub.com/content/3/1/21.full.pdf Rajaee, S., Cherkassky, L., Marcaccio, Jr., E. J., Carney, Jr., W. I., Chong, T., GarciaToca, M., \& Slaiby, J. M. (2014, February 18, 2014). Open Revascularization Procedures Are More Likely To Influence Smoking Reduction Than Percutaneous Procedures. Annals Of Vascular Surgery, 28(), 990-998. http://dx.doi.org/10.1016/i.avsg.2013.05.017 
Ronayne, RN, MScA, R., O’Connor, RN, PhD, A., \& Scobie, MD, FRCS(C), T. K. (1989). Smoking: a decision-making dilemma for the vascular patient. Journal of Advanced Nursing, 14(5), 647-652.

Tahiri, MD, M., Mottillo, MD, S., Joseph, PhD, L., \& Pilote, MD, MPH, PhD, L. (2012). Alternative Smoking Cessation Aids: A Meta-analysis of Randomized Controlled Trails. The American Journal of Medicine, 125(7), 576-584.

http://dx.doi.org/10.1016/j.amjmed.2011.09.028

Weinberger, A. H., Pilver, C. E., Mazure, C. M., \& McKee, S. A. (2014). Stability of smoking status in the US population: a longitudinal investigation. Addiction, 109, 1541-1553. http://dx.doi.org/10.1111/add.12647

Wu, P., Wilson, K., Dimoulas, P., \& Mills, E. J. (2006, December 11, 2006).

Effectiveness of smoking cessation therapies: a systematic review and metaanalysis. BMC Public Health, 6(300), 1-16. http://dx.doi.org/10.1186/1471-24586-300 